\title{
The Measurement of Low Temperatures
}

\author{
THE PROPERTIES AND APPLICATIONS OF \\ THE GOLD-IRON ALLOY THERMOCOUPLE
}

\section{Robert Berman}

Clarendon Laboratory, Oxford

In the rapidly advancing field of cryogenic engineering and research the accurate measurement of very low temperatures has become increasingly important. Alloys of gold with small amounts of iron have very large and reproducible thermoelectric powers in this range and are successfully used as the active limbs of sensitive and stable low temperature thermocouples.

The applications of low temperature technology are increasing rapidly. Liquid air and liquid oxygen have been used for some time, liquid air for rapid freezing of food and liquid oxygen as a compact and light source of the gas not requiring heavy steel cylinders for its transport. Liquid hydrogen is now a rocket fuel and represents an almost infinitely plentiful non-pollutant fuel for the future.

Liquid helium is the extreme coolant for purposes such as the reduction of "noise" in telecommunication systems, for example in the Goonhilly receiver, and more particularly, for maintaining superconductors in the non-resistive state. Many metals and alloys lose all electrical resistance at very low temperatures, and certain alloys can carry large currents in high magnetic fields without reverting to the normal, resistive, state. Electromagnets for fields up to $\sim 150$
$\mathrm{kG}$ with zero resistance and no power consumption can be constructed. Such magnets are used extensively in research laboratories all over the world and are beginning to have wider application. If fusion reactions become possible for power generation, it is likely that the magnets to contain the plasma will be superconducting. Small, light, ironfree electric motors and generators with superconducting windings can be built for many purposes, especially where the rate of rotation is not too high. A prototype motor for ship propulsion has been built and other applications can quite readily be envisaged (1).

A problem common to all research and development on these lines is accurate determination of the temperatures involved. If we wish to interpret the measured properties of matter, we must relate tem-

In the development of super. conductors for power engineering applications one of the most important parameters is the transition temperature at which they revert to the normal state. In this apparatus at the Central Electricity Research Laboratories at Leatherhead the sample is contained in a variable temperature capsule held just above the surface of liquid helium in the dewar in the foreground. The capsule temperature is monitored in the range 4.5 to 20K by a gold-iron/Chromel thermocouple

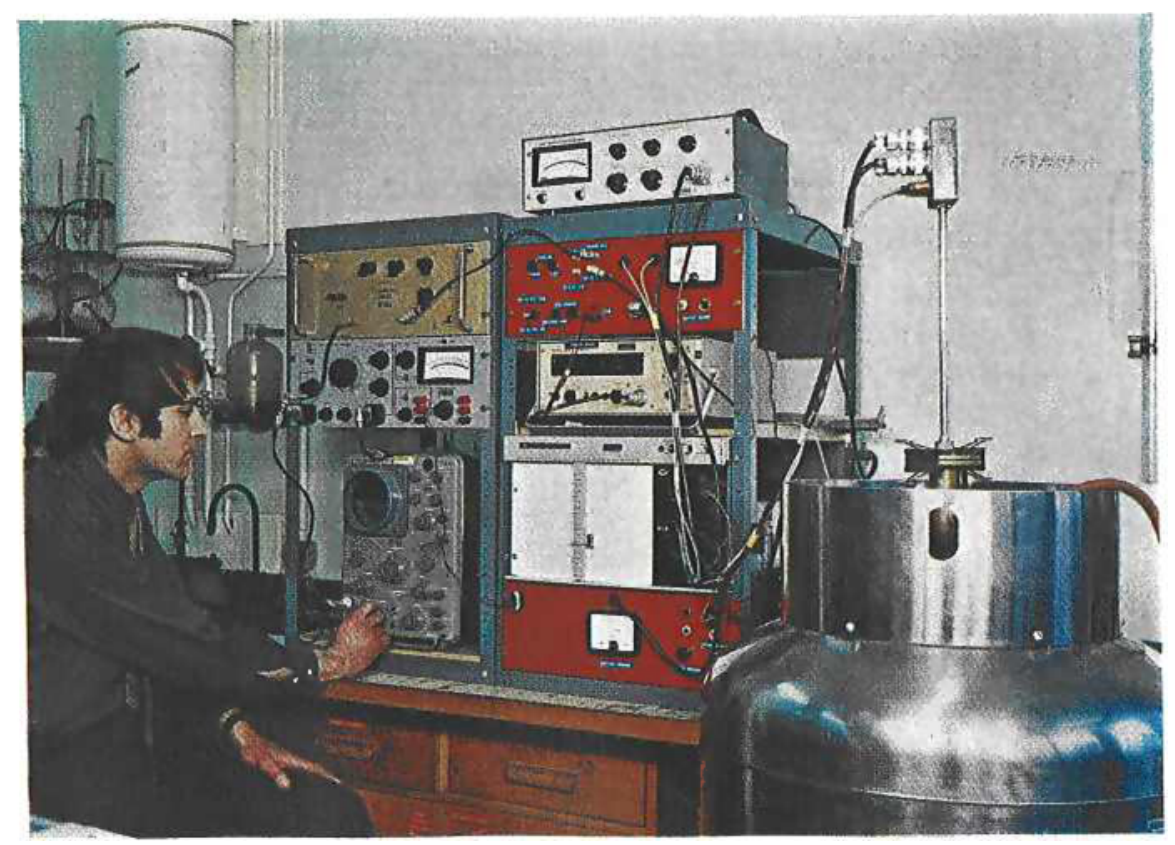


Parametric amplifiers, handling very weak signals at microwave frequencies as in satellite communications and in long distance telephony and television, need to be maintained at the lowest possible temperature to minimise interference by random noise. This helium refrigerator, one of a range specially designed by British Oxygen for this purpose, operates in the range 12 to $20 \mathrm{~K}$ using gold-iron thermocouples to monitor the temperature

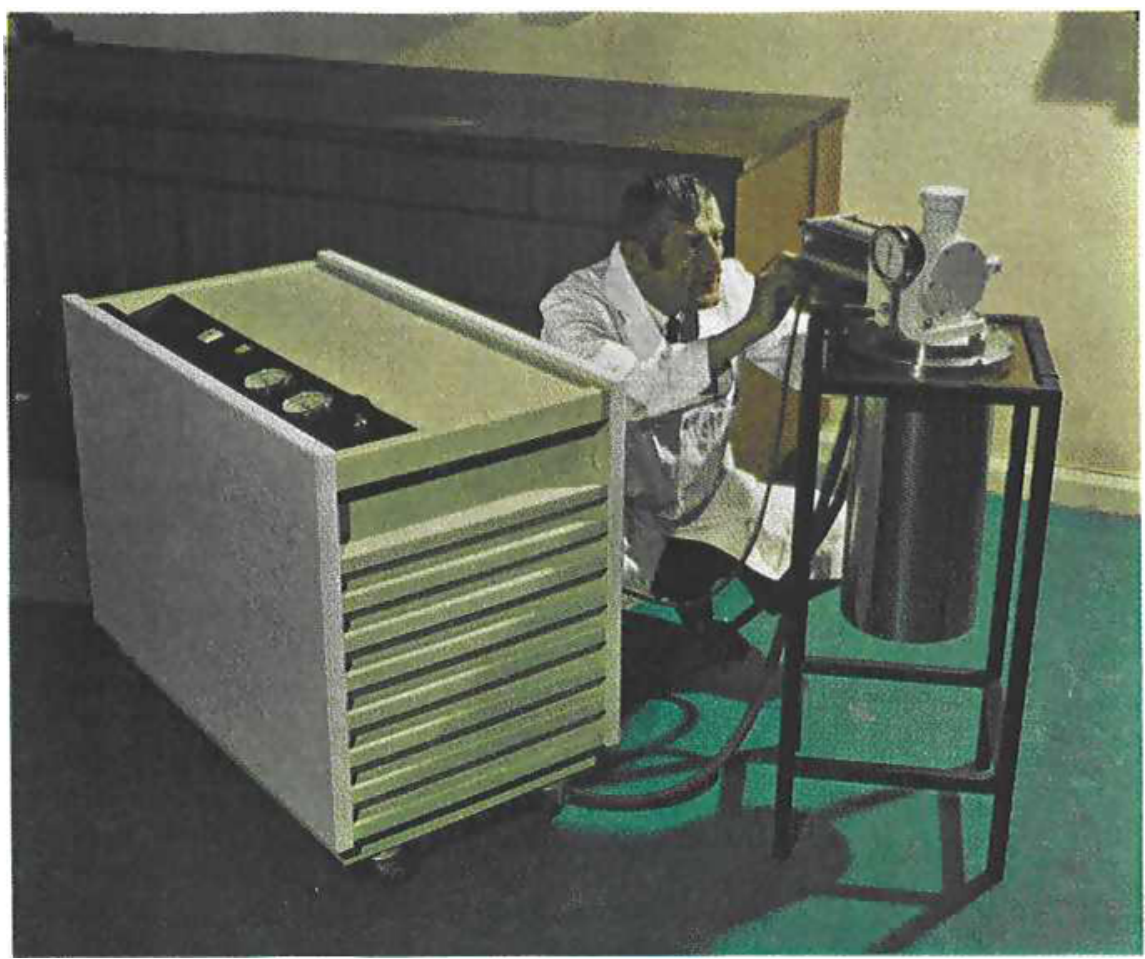

with either a platinum resistance or with a liquidvapour system. For example, high sensitivity may be required over a particular temperature range, or a low heat capacity for rapid response. Many practical thermometers depend on changes of electrical resistivity with temperature, since resistance changes can be measured with great accuracy by standard methods. To obtain large changes of resistivity at low temperatures impure materials are generally required, and the calibration depends on the exact amount of impurity added. Such thermometers must, therefore, be calibrated individually, or at least a few calibration points must be checked to derive the whole calibration from the known form of its variation from one sample to another.

Thermometers of suitably "doped" germanium are very stable over long periods of time and repeated temperature cycles, although errors of a few $\mathrm{mK}\left(10^{-3} \mathrm{~K}\right)$ can be introduced if they are not recalibrated during prolonged use. Carbon thermometers are usually in the form of commercial radio resistors consisting of small graphite particles embedded in a bonding material. Most have poorly reproducible calibrations, because the contacts among the graphite crystallites and between them and the electrical leads are altered on cycling the temperature.

It is sometimes more important to determine a small temperature difference accurately rather than an absolute temperature. It may then be more convenient to use a thermocouple, which is an instrument producing a voltage dependent on the difference in temperature of two junctions between 


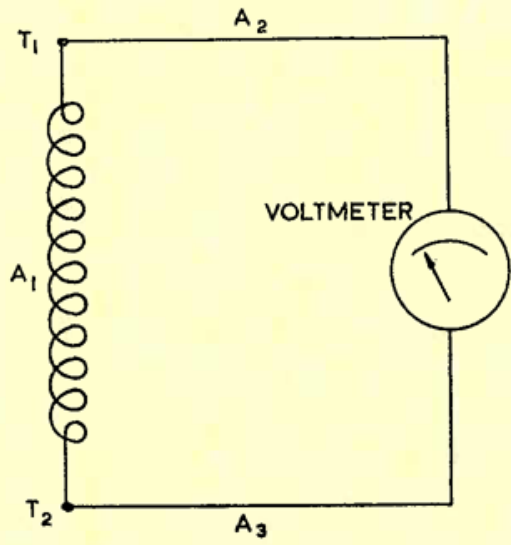

Fig. 1 The principles of thermocouple thermometry. $\mathbf{A}_{1}, \mathbf{A}_{2}$ and $\mathbf{A}_{3}$ are wires joined at temperatures $T_{1}$ and $T_{2} . A_{2}$ and $A_{3}$ are connected to the voltmeter

dissimilar conductors. Thermocouples also have the advantage of very low heat capacity as they consist essentially of two "points" at the junctions of thin wires. Thermocouples have not until recently been used much at low temperatures because the thermoelectric power (the e.m.f. produced for unit temperature difference) of most combinations obeys the Third Law of Thermodynamics in a docile way, decreasing gently to zero at absolute zero.

\section{Thermocouples}

If a temperature gradient is established along an electrical conductor there will, in general, be an e.m.f. set up between its ends. We might envisage making connections to a voltmeter as in Fig. 1 to determine this e.m.f., where $A_{1}$ represents the conductor with its ends at temperatures $T_{1}$ and $T_{2}$. Purely to shorten the discussion without affecting the principle to be illustrated, let us assume that the voltmeter is also at $T_{2}$. Because of the temperature difference along $A_{1}$ an e.m.f. is set up in it, but the same e.m.f. would be set up in the opposite direction in $\mathrm{A}_{2}$ if this is made of the same material as $A_{1}$. As there is no temperature difference along $A_{3}$ and no e.m.f. between its ends, the net effect is that no voltage will be measured by the voltmeter. No matter how the temperatures are juggled, no voltage will be recorded if all three arms are made of the same material.

If, however, arms $A_{2}$ and $A_{3}$ are made of a conductor in which the e.m.f. produced is different from that in $A_{1}$ for the same end temperatures, the voltmeter will measure the difference between these two e.m.fs. If one junction is held at a fixed temperature and the voltage $E(T)$ is measured while the temperature of the other junction is varied, the thermo- electric power, $S$, of the thermocouple is defined as $\mathrm{S}=\frac{\mathrm{dE}}{\mathrm{dT}}$ at temperature $\mathrm{T}$. At absolute zero $\mathrm{S}$ must be zero, and for many commonly used thermocouple combinations $S$ decreases monotonically with decreasing temperature and is so small below liquid hydrogen temperatures that the thermocouple becomes difficult to use. However, thermopower depends so delicately on details of the electronic structure, that no "anomalous" behaviour should come as a surprise! For example, even the sign of the e.m.f. for different conductors for fixed $T_{1}$ and $T_{2}$ is not always the same and for a given conductor may vary with temperature.

The anomalous behaviour of thermopower which is relevant here was discovered by Borelius and his co-workers in 1932 (2). The addition of small quantities of transition metals to a noble metal can enhance the thermopower enormously, and may even lead at low temperatures to a thermopower which increases with decreasing temperature and finally only tends rather slowly to zero.

For a number of years thermocouples, in which the active arm was made of gold plus two atomic per cent cobalt, were used quite extensively. However, the exact amount of cobalt which is in solid solution is sensitive to the thermal and mechanical treatment of the wire and the calibration was too irreproducible for many purposes. In addition, the sensitivity becomes rather small at liquid helium temperatures.

\section{Gold-Iron Thermocouples}

MacDonald and his collaborators (3) studied the thermopower of many dilute alloys at low temperature, and the material which has been developed for use

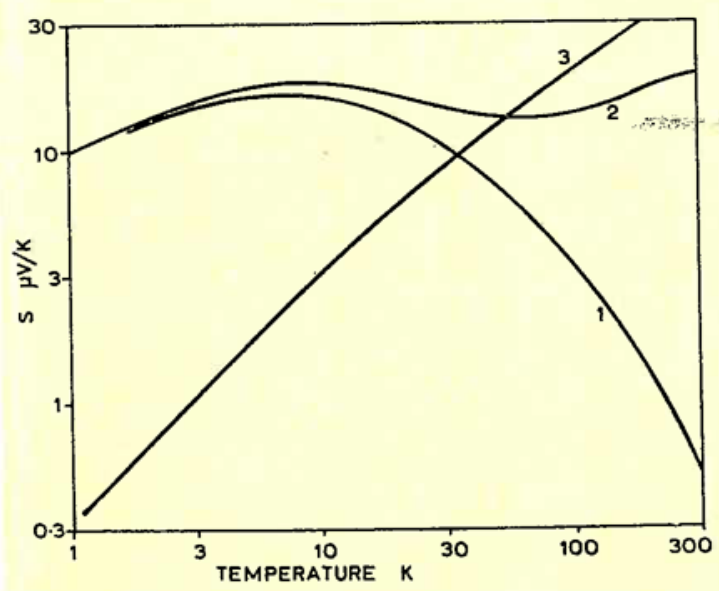

Fig. 2 Thermoelectric power of thermocouples: 1-Au+ 0.03at. $\%$ Fe vs. Ag+ $\mathbf{A g}$ at. $\%$ Au 2-Au+0.03at. $\%$ Fe vs. Chromel 3-Copper vs. Constantan 
The lower end of a cryostat, designed and built by Oxford Instruments, for X-ray diffraction studies of powder samples at all temperatures down to $4.2 \mathrm{~K}$. The main inner components of the cryostat, sample holder and radiation shield are made of copper plated with gold to provide the minimum absorptivity for radiated heat from the outer walls. The temperature is measured with a gold/ iron vs. Chromel themocouple, seen in the centre of the picture

in thermocouples is very pure gold with small quantities of iron (4). Figure 2 shows the thermoelectric power of two thermocouples with gold-iron arms and, for comparison, a copper-constantan thermocouple. The latter combination is commonly used at normal temperatures but its sensitivity falls gradually with decreasing temperature. For both curves 1 and 2 the gold contains 0.03 atomic per cent iron, but the second arm is different in the two thermocouples. For thermocouple 1 this arm is a standard alloy of silver plus 0.37 atomic per cent gold (known as silver "normal"), which is relatively "inert", so that the thermopower shown represents almost entirely the thermopower of the Au-Fe alloy. The second arm for thermocouple 2 is Chromel, an alloy of 90 per cent nickel, 10 per cent chromium used in high temperature thermocouples. The thermopower of Chromel is of opposite sign to that of the gold-iron and starts to become appreciable above $\sim 20 \mathrm{~K}$, where the thermopower of gold-iron begins to fall off. The thermopower of the combination is, therefore, the sum of the individual thermopowers, with the gold-iron being the dominant partner at low temperatures and Chromel at high temperatures. This results in a thermocouple with sensitivity not less than $10 \mu \mathrm{V} / \mathrm{K}$ at all temperatures above $1 \mathrm{~K}$.

If measurements are confined to temperatures below about $15 \mathrm{~K}$, the thermopower cannot be enhanced appreciably, and it is sometimes advantageous to use a superconductor as the second arm. Superconductors have no thermoelectric power, so that spurious e.m.f.s, which give rise to experimental difficulties when using thermocouples, do not arise in them.

The large thermopower of gold-iron is due to the scattering of the conduction electrons in gold by the magnetic moments of the iron. If the iron concentration is "high", interactions between the magnetic moments reduce the thermopower at the lower temperatures. If the concentration is "low", then at the higher temperatures the magnetic scattering is dominated by the normal scattering of electrons by lattice vibrations, and the anomalous thermopower is suppressed (5). The starting gold must be very pure so that electron scattering by

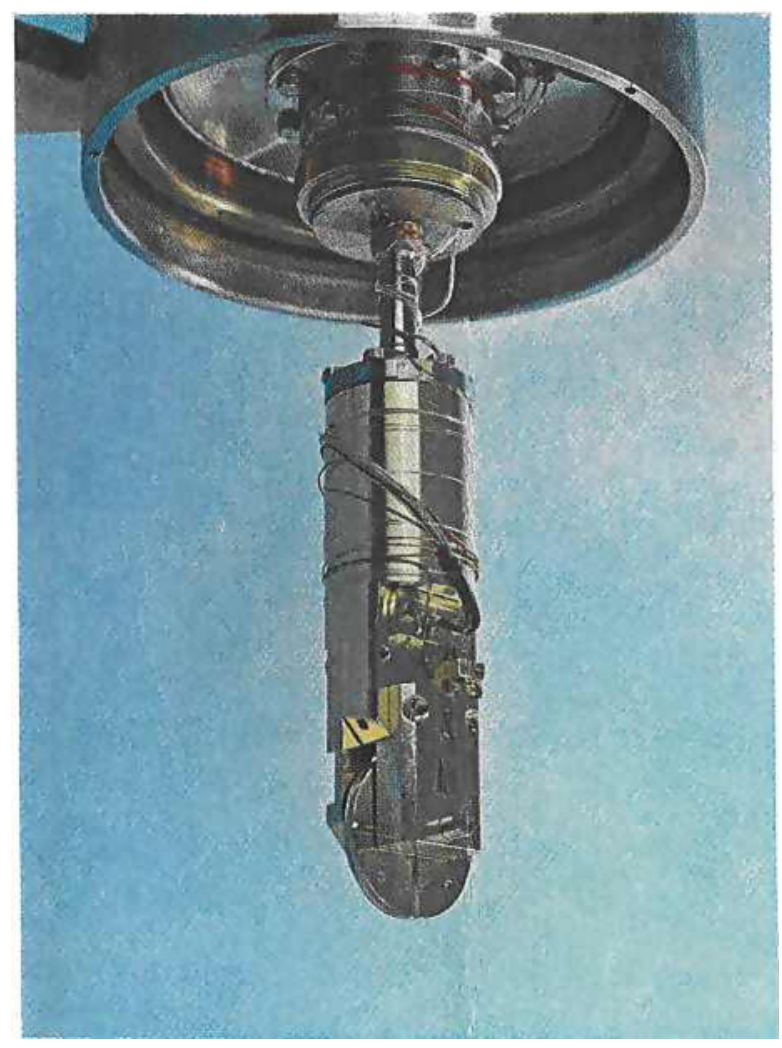

ordinary impurities should be small. For general purposes one wants high sensitivity from below $1 \mathrm{~K}$ to about $20 \mathrm{~K}$ (above this temperature the second arm can be chosen to enhance the thermopower), with the sensitivity being not too dependent on iron concentration. This aim is achieved by an alloy containing of the order of 0.05 atomic per cent iron. The wire produced by Johnson Matthey is made from spectroscopically pure gold with 0.03 atomic per cent iron, while in the U.S. the standard material contains 0.07 atomic per cent iron.

\section{Practical Considerations}

Apart from having adequate sensitivity, a thermometer must have sufficient reproducibility for the purpose to which it is being put. Because of its role in IPTS-68, a great amount is known about the variability of platinum resistance thermometers, but there is no comparable corpus of experience with low temperature thermocouples. However, from the systematic experiments of Sparks and Powell (6), together with some work of Rosenbaum (7) and of ourselves, we can derive some picture of the range of reproducibility of calibration likely to be encountered when using gold-iron thermocouples. A summary of these experiments is given here.

(a) A gold-iron vs. superconducting niobium thermocouple was calibrated between 1 and $4 \mathrm{~K}$ before and after a series of about forty experiments, 
Calibration of four Gold-0.03 atomic per cent Iron vs. Chromel Thermocouples with one Junction at the Helium Boiling Point

\begin{tabular}{|c|c|c|c|c|}
\hline \multirow{2}{*}{$\begin{array}{l}\text { Temperature } \\
\text { of variable } \\
\text { junction } \\
\text { (K) }\end{array}$} & \multicolumn{3}{|c|}{$\begin{array}{l}\text { e.m.f. (microvolts) } \\
\text { single batch of alloy }\end{array}$} & \multirow{2}{*}{$\begin{array}{l}\text { Earlier } \\
\text { batch of } \\
\text { alloy }\end{array}$} \\
\hline & Reel 1 & Reel 9 & Reel 17 & \\
\hline 4.21 & 0.0 & 0.0 & 0.0 & 0.0 \\
\hline 10.11 & 82.0 & 81.4 & 82.2 & 88.8 \\
\hline 20.15 & 231.9 & 231.6 & 232.4 & 249.7 \\
\hline 30.32 & 374.7 & 374.6 & 375.6 & 399.7 \\
\hline 39.96 & 503.7 & 503.9 & 505.0 & 532.8 \\
\hline 50.11 & 639.3 & 639.5 & 640.5 & 671.2 \\
\hline 60.29 & 778.4 & 779.0 & 779.8 & 812.7 \\
\hline 69.41 & 907.7 & 908.3 & 908.9 & 943.6 \\
\hline 80.00 & 1063.2 & 1064.0 & 1064.4 & 1100.7 \\
\hline 90.44 & 1222.8 & 1224.2 & 1225.1 & 1262.0 \\
\hline 99.78 & 1370.4 & 1371.9 & 1372.8 & 1410.7 \\
\hline
\end{tabular}

Calibrations by Cryogenic Calibrations, Pitchcott, Aylesbury, Buckinghamshire, England.

The reels each contained 3 metres of wire, so that there are about 50 metres between reels 1 and 17 .

each of which entailed cooling from room temperature and maintaining the apparatus for at least twelve hours at the low temperatures before warming up again. No change in calibration as great as 0.5 per cent was found over the two-year period involved. Our general experience is in line with this observation on the effect of temperature cycling alone. (b) Sparks and Powell have kinked and stretched pieces of wire and measured the e.m.f. of thermocouples made from a deformed with an undamaged length. This treatment changed the calibration so little that if the deformed wire were part of a gold0.03 per cent iron vs. Chromel thermocouple with one junction at the ice point, then all temperatures deduced down to $4 \mathrm{~K}$ would be unchanged within $0.1 \mathrm{~K}$.

(c) Comparisons between adjacent pieces of wire taken from the same reel suggest that in general the calibrations do not differ by more than 1 per cent, but specimens taken from three different reels drawn from one batch of alloy, measured by Ricketson (8) showed very remarkable agreement, as shown in the table. Results are shown at about $10 \mathrm{~K}$ intervals and only the results below $100 \mathrm{~K}$ are given, because above this temperature the Chromel contributes more than half the measured e.m.f., so that any irreproducibility in the gold-iron is relatively less important.

It appears that the more usual variability between reels from one batch of alloy is similar to differences between different batches and between wires produced by different manufacturers. At the lowest temperatures, the sensitivity of the gold-iron wire is so dependent on iron concentration that larger variations in calibration must be expected. Goulder (9) has recently calibrated, between 0.4 and $2 \mathrm{~K}$, four pieces of gold- 0.03 per cent iron wire used at different times in different apparatus in this laboratory, covering a period of about six years in manufacture. The sensitivities are shown in Figure 3.

Equipment for the calibration of low temperature sensors between 2 and $273 K$ has been specially set up by Cryogenic Calibrations. The sensors are placed in closely fitting holes in the copper block at the bottom of the low temperature apparatus (shown dismantled) on the right, which is contained in a dewar holding liquid nitrogen or helium. On the left is an accurate temperature controller and a controlled D.C. output for the heater coil on the sensor block and a temperature controlled box for resistance and voltage standards. On the table is a Cryobridge automatic balane. ing potentiometer for measurement with $25 \mathrm{~Hz}$ current, and behind the operator a Tinsley Staubomatic potentiometer for D.C. measurements, with galvanometer and current control devices above

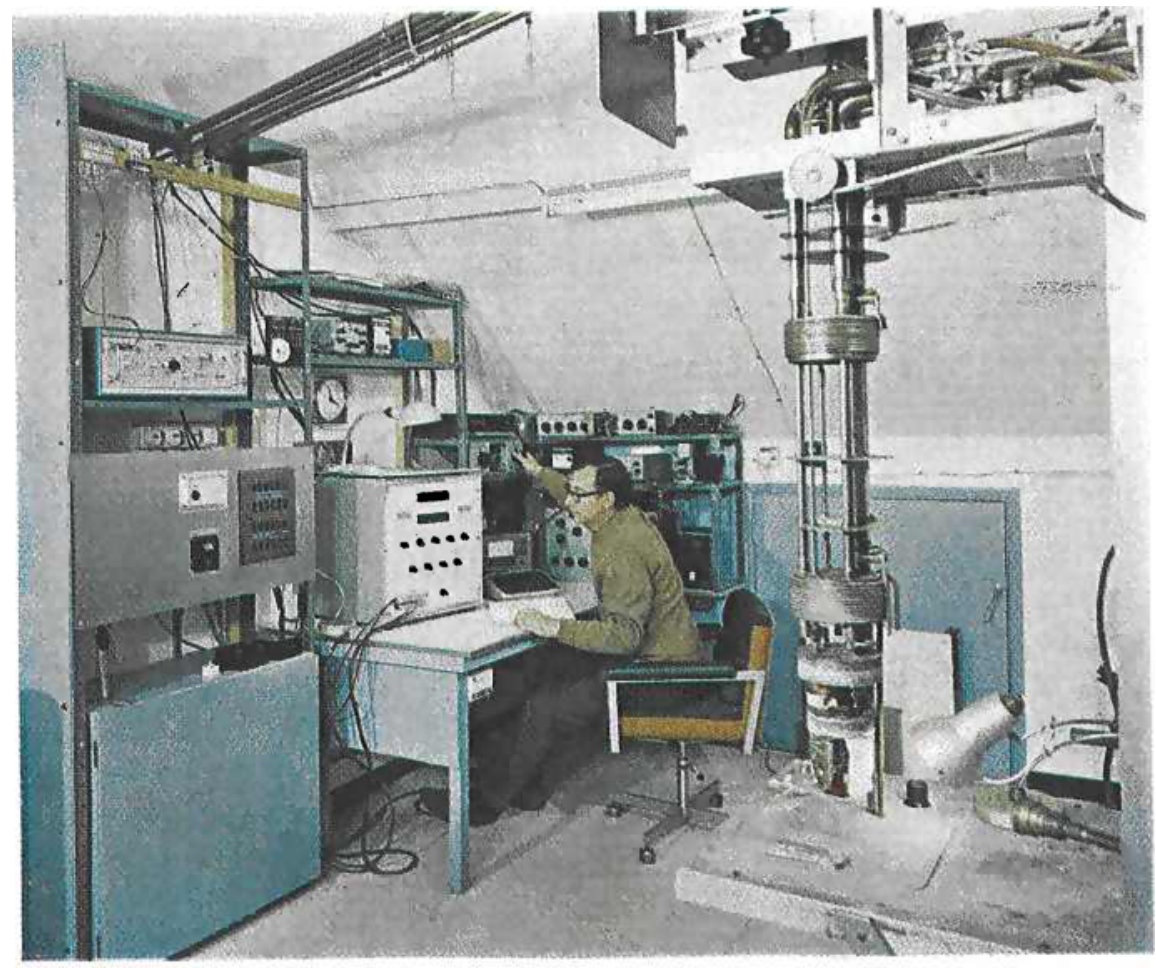




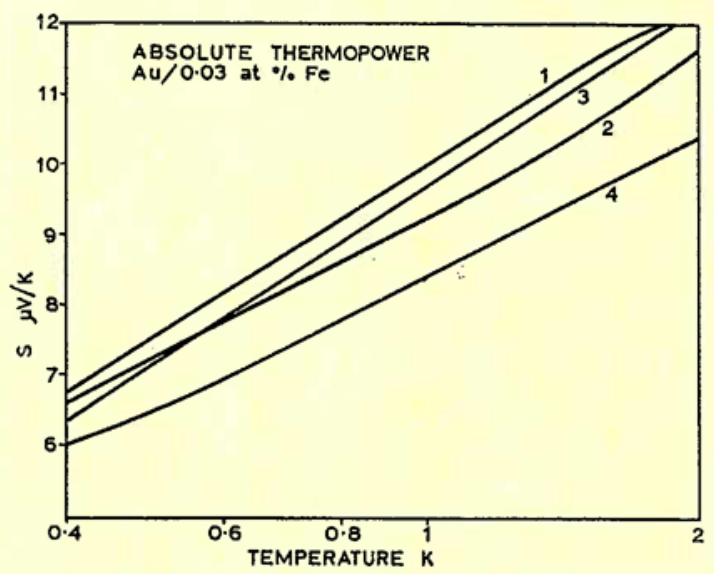

Fig. 3 Calibration of three $\mathrm{Au}+0.03$ at. $\%$ Fe vs. superconductor thermocouples below 2K. Approximate years of manufacture:

1-1966, 2-1967, 3-1969, 4-1970

From all these experiments we may conclude that the calibration of a gold-iron wire used in a thermocouple is exceedingly stable during repeated temperature cycling and is little affected by normal mechanical handling. As the sensitivity down to $\sim 1 \mathrm{~K}$ is comparable with that of thermocouples in common use at normal temperatures, there are many low temperature applications where they can be conveniently used, employing standard electrical measurement techniques. The differences between different batches of wire are sufficient to require checking of the calibration at a few conveniently realised fixed points if a temperature difference is required to be known to better than about 1 per cent. However, all the calibrations seem to differ from one another in a smooth manner so that adjustments are not difficult to make.

\section{References}

1 See, for example, J. K. Hulm, Cryogenics, 1972, 12, 472

2 G. Borelius, W. H. Keesom, C. H. Johansson and J. O. Linde, Proc. Kon. Akad. Amsterdam, 1932, 35, 10

3 W. B. Pearson and I. M. Templeton, Canad. F. Phys, 1961, 39, 1084; D. K. C. MacDonald, W. B. Pearson and I. M. Templeton, Proc. Roy. Soc., 1962, A266, 161

4 R. Berman and D. J. Huntley, Cryogenics, 1963, 3, 70 R. Berman, J. C. F. Brock and D. J. Huntley, Cryogenics, $1964,4,233$

5 R. Berman and J. Kopp, F. Phys. F: Metal Phys., 1971, 1, 457

6 L. L. Sparks and R. L. Powell, Measurements and Data 1967, 1, 82; F. Res. Nat. Bur. Standards, 1972, 76A, 263

7 R. L. Rosenbaum, Rev. Sci. Instrum., 1968, 39, 890; ibid., 1969, 40, 577

8 B. W. A. Ricketson, Private communication

9 D. P. Goulder, Private communication

\section{A Low Temperature Resistance Thermometer}

The well-known platinum resistance thermometer - widely used in industry and accepted for part of the International Temperatures Scale over the range from $13.8 \mathrm{~K}$ to the ice point-has disadvantages at very low temperatures in that the resistance to be measured is then small, while it is inconvenient to measure resistances with values spread over three decades.

The addition of small amounts of transition metals to gold has an anomalous effect on its resistivity at low temperatures, and on this basis a new type of cryogenic resistance thermometer has been developed by Degussa of Hanau, West Germany. This employs a 0.4 per cent manganese-gold alloy which has proved to be entirely stable and can be drawn into fine wire.

The addition of this small amount of manganese to gold is dramatic in that the resistance of a sensor that is $50 \mathrm{ohms}$ at the ice point falls to $25 \mathrm{ohms}$ at helium temperatures rather than to $0.01 \mathrm{ohm}$ with pure gold. Most of this additional resistance is temperature independent, but the alloy still has a positive slope of resistance against temperature from a value that is probably as low as $0.1 \mathrm{~K}$.

The detection voltage for any given accuracy of measurement is dependent on the maximum current that may be passed through the sensor without causing the wire to overheat appreciably and on the value of the fractional sensitivity $\mathrm{dR} / \mathrm{RdT}$. The maximum current for this new thermometer in any particular thermal environment is likely to be similar to that of platinum thermometers of the same resistance and construction. The fractional sensitivity is better than that of the nickel-manganin strain gauge type of sensor and appreciably the same as that of the gallium-arsenide diode. It is at its highest around $2 \mathrm{~K}$, but falls rapidly by nearly a magnitude between 10 and $15 \mathrm{~K}$.

Linearisation, an important electronic process that allows a display of temperature from sensor measurement, should be comparatively easy between $40 \mathrm{~K}$ and the ice point as the sensitivity alters by only about 10 per cent.

If the reproducibility of this sensor-marketed under the name Keltip-proves to be high with time and thermal cycling, a very useful new instrument will have become available for both industrial and laboratory use, particularly where millikelvin accuracy is not essential but where simple and multiple presentation of temperature is required.

B. W. A. R. 